\title{
Variations
}

Variations

Revue internationale de théorie critique

16 | 2012

Tahrir is here !

\section{Un espace public néocapitaliste ? Habermas, un demi-siècle après}

\section{Stéphane Haber}

\section{OpenEdition}

\section{Journals}

Édition électronique

URL : http://journals.openedition.org/variations/204

DOI : 10.4000/variations.204

ISSN : 1968-3960

\section{Éditeur}

Les amis de Variations

\section{Édition imprimée}

Date de publication : 16 janvier 2012

\section{Référence électronique}

Stéphane Haber, «Un espace public néocapitaliste? Habermas, un demi-siècle après », Variations [En ligne], 16 | 2012, mis en ligne le 20 février 2012, consulté le 20 avril 2019. URL : http:// journals.openedition.org/variations/204 ; DOI : 10.4000/variations.204

Ce document a été généré automatiquement le 20 avril 2019.

Les ami•e•s de Variations 


\title{
Un espace public néocapitaliste? Habermas, un demi-siècle après
}

\author{
Stéphane Haber
}

\section{NOTE DE L'ÉDITEUR}

Première publication sur www.theoriecritique.com, « Tahrir is here ! ", 16 janvier 2012, pp. 79-92

1 Strukturwandel der Öffentlichkeit ${ }^{1}$, paru il y a cinquante ans, n'est pas d'abord un livre d'histoire, mais un livre politique. Comme on le sait, il s'agissait essentiellement pour son auteur de réveiller un concept de démocratie que le nazisme et le stalinisme avaient fait entrer dans un sommeil dogmatique, en installant au-devant de la scène des idées une opposition entre «totalitarisme» et "démocratie» qui, dans les faits, contribuait beaucoup à figer une conception étroitement électorale-délégataire-partidaire de cette dernière. En termes contemporains, on pourrait dire en bref qu'il s'agissait, pour Habermas de «relancer la critique sociale » à partir d'un programme intellectuel et politique centré autour d'une conception exigeante et non-conventionnelle de la démocratie. Il s'agissait de montrer que la démocratie a plus à avoir avec une société civile politiquement vigilante, mobilisée, inquiète du sens du présent historique et de son avenir, qu'avec les processus électoraux ou avec le triste fonctionnement de la machinerie représentative étatique et para-étatique, laquelle, en fait, encourage la dépolitisation. Clairement, l'analyse historique représentait donc, dans l'ouvrage de 1962, un détour. Elle devait établir qu'une culture post-traditionnelle peut, en principe, se stabiliser autour d'authentiques pratiques démocratiques en arguant du fait qu'elle s'est à un certain moment approchée de ce modèle, et que, en tout cas, la constitution de la culture moderne s'est en partie traduite par un basculement au terme duquel la reproduction de contenus traditionnels a cédé sa place à l'invention d'éléments qui exprimaient sous une forme ou une autre une compréhension de soi proprement historique. Mais Habermas a effectué ce détour avec un tel sérieux, il s'est tellement pris 
au jeu, en quelque sorte, qu'une appréciation contemporaine de l'ouvrage est tout à fait fondée à partir de là.

Parmi toutes les évaluations possibles, les travaux historiques ont souvent convergé vers un même genre de conclusions ${ }^{2}$. Même partiellement contrôlée, la tentation peut-être inévitable qu'exerce le modèle du récit à la courbe nette, dans le style "émergence, apogée puis déclin », ou bien "grandeur et décadence ", s'est révélée trop forte chez Habermas. On sait que l'âge des Lumières aurait ainsi été, selon lui, celui de la constitution d'une forme rationnelle nouvelle, celle d'un public cultivé et discutant, commençant à devenir capable de faire plier la logique de la domination publique. Et depuis, l'histoire politique et culturelle de l'occident aurait plutôt été animée, affirme-til, par des forces régressives qui cherchèrent et parvinrent souvent à limiter ou à distordre cette puissance sociale d'un nouveau genre. Or, face à l'attraction exercée par ce modèle narratif, tout se passe comme si la très claire conscience qu'avait Habermas du caractère encore intrinsèquement élitiste et bourgeois de l'opinion publique à l'époque des Lumières n'avait finalement pas fait vraiment le poids, n'avait pas vraiment compté pour les hypothèses de base de son ouvrage. Il semble qu'une connaissance même superficielle des travaux historiens portant sur la période antérieure au 19e siècle donne aujourd'hui suffisamment de motifs pour mieux résister à cette attraction ${ }^{3}$. L'existence d'une culture dynamique de la discussion critique, alimentée, d'une part, par des pratiques telles que la lecture, influençant, d'autre part, la vie des idées comme l'univers politique, cela a pu prendre des formes très nombreuses, parfois indécises, cela a emprunté des itinéraires sinueux, parfois inattendus. L'une des erreurs de Habermas, la principale, sans doute, peut-on affirmer, consiste à avoir prêté à certains faits sociaux une cohérence que l'on avait encore, à l'époque où il écrivait, l'habitude d'attribuer, à tort, aux « Lumières » européennes comme mouvement de pensée 4 .

\section{Sur le fond, quelles alternatives critiques?}

3 Le principe philosophique de ce biais interprétatif qui marque le livre de 1962 (et qui n'est pas totalement effacé dans la "préface » partiellement autocritique de la réédition de 1990) est facile à isoler. Il tient à une croyance fascinante et, sans doute, pas entièrement fausse, bien qu'ici malheureusement prise au pied de la lettre : la croyance selon laquelle, quand des gens se mettent à parler selon des règles qui sont même approximativement de la discussion digne de ce nom (centralement : liberté et égalité de parole), une nouvelle sphère, celle de l'autonomie, s'ouvre dans la vie sociale, des principes clairs et nets apparaissent enfin au-dessus de la masse confuse des habitudes et des règles qui structurent la vie sociale ; certaines conditions de l'émancipation sont fermement posées. Ce principe d'interprétation anticipe bien évidemment, dans l'élément sociologicohistorique, les conclusions d'une "pragmatique universelle » et d'une "éthique de la discussion ", que Habermas n'explicitera que plus tard dans sa carrière. Il en préfigure aussi les difficultés. Car il est aisé de voir que l'intuition selon laquelle les normes de la discussion dignes de ce nom sont, en quelque sorte, indépendantes et supérieures à toutes les valeurs comme à tous les principes de la vie éthique conduit à une série de paradoxes. Elle conduit par exemple à l'idée selon laquelle entre, d'une part, la reconnaissance de l'honnêteté comme vertu ordinaire dans la vie et, d'autre part, la conviction qui veut qu'une discussion bien menée passe par une certaine forme de respect des personnes en présence ou par une attention sincère à leurs arguments, le rapport serait médiat et non 
pas direct. Une fois que l'on s'installe dans l'élément transcendantal, dans les métanormes hypothétiques de la discussion vraie, la redescente vers le réel, vers la vie, devient difficile, en tout cas, méthodologiquement problématique.

Pourtant, il est clair qu'un espace public ne fonctionne pas tout seul ni selon des lois autonomes, bien qu'il puisse, si l'on y tient, être vu comme une manifestation de l'autonomie humaine. Il est certes vrai que, avec l'expression langagière, et surtout celle qui s'oriente vers la discussion, la vie s'engage dans des aventures nouvelles et prometteuses. Il y a bien quelque chose qui se passe ${ }^{5}$. Mais elle ne peut le faire qu'en s'appuyant continument sur ce qui la précède et lui reste extérieur - quand bien même ce serait cet événement qui conférerait un sens unitaire saillant à ces éléments préexistants. La dépendance contextuelle du sens des énoncés que nous produisons quotidiennement constitue la manifestation à la fois la plus limpide et la plus fondamentale de cette relation' ${ }^{6}$. C'est elle qui, par exemple, nous incite à penser, si l'on franchit sans façons quelques-unes des médiations qui doivent nous rapprocher du concret sociologique, que les normes de la discussion ne s'articulent et ne produisent d'effets qu'à certaines conditions, qu'elles ont besoin de schèmes et d'appuis extérieurs. On peut dire qu'elles ont, en particulier, besoin de dispositions éthiques (par exemple des vertus gravitant autour de la générosité et de l'attention), d'une infrastructure économique marquée par un tissu d'échanges justes et de pratiques consistant à donner, à recevoir, à partager, seules aptes à soutenir dans la durée les échanges communicationnels. On peut dire aussi qu'elles ont aussi besoin de s'articuler à des processus d'enquête, au sens non seulement de la confrontation intelligente des positions en présence, mais d'un travail collectif de mise à l'épreuve et d'évaluation concrète des propositions, selon les contraintes et les contextes ${ }^{7}$. Sous ses formes les plus autonomes, la pratique langagière-discursive, dont l'intervention dans l'espace public constitue une modalité, reste donc encore une pratique, une manière faillible de tracer des chemins dans le réel, qui reste soumise aux mêmes conditions que les autres pratiques, leur empruntant souvent des éléments, vivant au milieu d'elles. C'est sur cet arrière-plan philosophique contextualiste que se détache l'idée selon laquelle l'espace public digne de ce nom n'est pas la réalisation même approximative du modèle d'une discussion éthérée entre esprits purs, déliés de leurs attaches mondaines (alors que, par certains côtés, on n'a pas tort de prêter à Habermas une telle approche hyperidéalisante).

5 Dans ces conditions assurément restrictives, y a-t-il encore un sens à investir d'une signification essentielle et unitaire les phénomènes relevant de ce que l'on appelle l'öffentlichkeit, "l'espace public», que ce soit en historien ou en philosophe? Pragmatiquement, politiquement, l'intuition paraît y conduire. Ainsi, une limitation injustifiable de la liberté d'expression, une manipulation médiatique, une stratégie de communication mensongère orchestrée par une organisation politique ou économique, la pauvreté des réseaux permettant la communication publique, etc., nous paraissent porter atteinte à une des exigences profondes de la société démocratique en général, et pas seulement à la liberté individuelle ${ }^{8}$. Quelque chose du tissu social propre à la démocratie semble alors se déchirer. Bref, certaines formes de pathologies sociales nous ramènent au thème de l'espace public et à son importance, que le détour par la philosophie du langage pouvait conduire à relativiser. Mais la manière dont cette intuition sociologico-politique apparemment assez mal dégrossie peut être judicieusement défendue aujourd'hui n'est pas simple à définir. 


\section{Un nouveau rapport à l'espace public}

6 À ce propos, notre thèse, d'inspiration matérialiste, sera la suivante: par rapport à l'époque où Habermas développait ses premières idées, ce qui a changé les choses, c'est surtout (d'autres éclairages sont possibles) que le capitalisme contemporain entretient une relation bien plus complexe et, globalement, bien plus intime (car, en gros, non médiatisée par l'État social) à l'espace public. C'est-à-dire que le néocapitalisme a instauré un relation nouvelle à cet étage de la société civile où s'élabore, dans un milieu d'échanges animés qui est centralement d'ordre langagier (mais pas uniquement), une sorte de conscience de soi historique et collective, la conscience de ce que nous sommes, du monde dans lequel nous vivons et de ce que nous voulons (en) faire.

7 Du point de vue normatif, cette hypothèse de la nouvelle intimité suggère assurément qu'un espace public authentique ne saurait être présenté aujourd'hui comme quelque chose d'absolument différent de ce que promeut le néocapitalisme communicationnel et interactif (ainsi que les formes d'exercice du pouvoir qui l'accompagnent). Un meilleur espace public ne pourrait consister que dans le résultat réel-possible de processus irréductiblement divers, capables de dessiner un ensemble précaire; il ne pourrait consister que dans le résultat émergeant d'une série de corrections, de détournements opérés sur l'existant, d'inventions de contenus plus difficilement assimilables par le système, de nouvelles formes informatives, délibératives ou critiques. Une alternative historique deviendrait pensable si les contre-forces de ce genre devenaient capables d'acquérir une influence telle que des dynamiques profondes et cumulatives de changement social et culturel soient capables de se mettre en place.

8 Du point de vue ontologique, on se trouverait, avec l'hypothèse de l'intimité, en une certaine affinité avec l'idée selon laquelle, dans le langage, l'impureté (par exemple sous la figure de l'intention instrumentale sur surajoutant l'intention communicationnelle, de l'ambigüité, de l'incompréhension et du malentendu) est aussi constante qu'originelle. C'est même là un corrélat incontournable de la position contextualiste qui a été évoquée plus haut: non seulement il faut un contexte pour que le sens soit compris et que la communication dévoile son potentiel, mais encore les bons contextes sont aussi rares que fragiles - ce qui n'est certes pas dramatique en soi. Que le capitalisme, image paradigmatique de ce qu'est une puissance aliénante, puisse à ce point s'agréger et s'accrocher à l'élément de la vie publique, s'associer au langage de la discussion, voilà qui est riche d'enseignements. Une des conclusions que l'on peut tirer de telles proximités est que faire l'histoire de l'espace public (un objet de recherche dont l'importance reste évidente) ne peut consister à dévoiler la constitution miraculeuse d'une poche de résistance ou encore les débuts héroïques d'une conquête de l'autonomie collective, comme dans le livre de Habermas. Les profondes ambivalences du présent historique, où le capitalisme et l'espace public se lient intimement sans se perdre, rendent peut-être plus sensibles aux situations passées, au fait que l'émergence de l'espace public ne saurait être pensée comme un événement miraculeux, irréversible, image de l'émancipation humaine en marche. Il est normal et inévitable qu'elle soit toujours enchâssée dans des relations de pouvoir ou dans des contraintes systémiques.

Nous nous voyons en tout cas obligés de nuancer la conception habermassienne d'une colonisation contemporaine de l'espace publique, conception selon laquelle les forces anti-publiques investissent quelque chose qui existait avant elles et pourrait sans 
problème persister sans elles. Le "capitalisme avancé » se manifeste aujourd'hui moins sous la forme d'une continuation des bonnes veilles techniques de corruption, de manipulation et de répression - idée dont Habermas n'est pas loin - que comme une manière plus subtile de tenter d'investir l'espace public pour bénéficier de sa puissance, de s'immiscer dans son fonctionnement et, pratiquement, de contribuer à diriger son développement: en un mot d'essayer, en brouillant les frontières, tout à la fois de l'exploiter, de le produire et de l'intégrer à son fonctionnement. Une sorte de grand principe de continuité domine donc. Mieux encore : dans la mesure où cette tendance est globalement couronnée de succès, on peut dire que le capital se régénère au moins partiellement grâce à l'espace public, s'installant décidemment, pour cela, dans ses marges, ce qui conduit finalement à brouiller les frontières entre l'intérieur et l'extérieur et à renoncer, du coup, à la métaphore dualiste de la " colonisation ", chère à Habermas. Le capital se porte spontanément vers cette zone trouble entre public et non-public, dont l'existence, sur le long terme, modifie les deux termes de la relation, au lieu de continuer à travailler, comme autrefois, à l'écart, dans la pénombre des espaces non-publics au sens habermassien. Autrefois désertiques, les zones frontalières entre ce que Habermas, dans ses ouvrages ultérieurs, appellera « le système » et « le monde de la vie » se trouvent ainsi désormais peuplées d'entités aussi ambiguës que mobiles ${ }^{9}$. C'est cela qui, pour nous, symbolise paradoxalement, d'un point de vue historique, l'autonomie irréductiblement relative de l'espace public.

\section{La relation intime entre capitalisme et sphère publique}

Les manifestations de la relation intime qui unit désormais capitalisme et espace public peuvent être réparties en trois catégories. (1) Un certain nombre de phénomènes illustre la nouvelle centralité économique et culturelle des techniques d'aménagement de l'espace, de transport et de communication, techniques qui exercent une grande influence sur la définition même de l'espace public: le rapport, ici, semble être de proximité plus que d'opposition. (2) À un autre niveau, le déplacement du centre de gravité de la production vers l'immatériel transforme les rapports entre l'univers économique et l'öffentlichkeit, entre production et langage: il se produit parfois une sorte de synergie entre les deux éléments, qui en viennent, dès lors, à s'interpénétrer ${ }^{10}$. (3) Enfin, à un autre niveau encore, l'expansion de la puissance économique de l'« industrie de la culture » modifie le sens même de la culture, entendue comme cette ressource qui alimente l'espace public en images et en discours tout en bénéficiant des processus qui s'y développent : en fait, la vitalité de l'industrie culturelle accroit celle de l'espace public en même temps qu'elle la distord. Ici, la distance entre capitalisme et espace public semble pratiquement s'évanouir.

11 1. Le capitalisme est-il nécessairement étranger à l'espace public et à la raison publique ? Est-il par nature hostile à l'existence de lieux dans lesquels, animés par l'expression et la confrontation des expériences et des idées, se constitue collectivement, intersubjectivement, une conscience historique et critique de soi à l'échelle de la société ? À première vue, on pourrait le croire situé au côté des influences occultes, des mécanismes anonymes, irrésistiblement voué à chercher à contrôler ou à perturber le fonctionnement de l'espace public en fonction d'intérêts privés. Il faut admettre que les approches qui déploient, d'une façon ou d'une autre, cette intuition restent puissamment éclairantes ${ }^{11}$. M. Davis montre ainsi, à propos du cas de l'aménagement urbain de Los 
Angeles $^{12}$, à quel point les politiques néolibérales ont visé, en tout cas ont abouti, à la fin du siècle dernier, à privatiser ou à éliminer les lieux politiques (jardins, rues, établissements et équipements collectifs) sous la double pression des intérêts commerciaux et d'un sécuritarisme forcené ${ }^{13}$. Comme si toute expansion du capitalisme devait se traduire par une sorte d'atrophie de la place réservée à la civilité et à la sociabilité propres aux «lieux publics», infrastructures et symboles concrets de «l'espace public » (Öffentlichkeit) ${ }^{14}$. En s'appuyant sur les travaux de W. Benjamin, on peut cependant observer l'existence d'une tendance complémentaire qui ne va pas forcément dans le même sens : la tendance du capitalisme à investir et même à susciter des lieux publics, à réaménager à partir de ses propres exigences l'univers urbain et ses espaces caractéristiques ${ }^{15}$. Benjamin décrivait ainsi les «passages couverts » du Paris du 19e siècle comme l'expression historiquement originale d'un phénomène que Marx avait nommé «fétichisme de la marchandise »: dans les phases avancées du capitalisme, ce fétichisme se trouverait, en quelque sorte, pris en charge réflexivement, activement, par le capitalisme (on penserait aujourd'hui aux centres commerciaux, à l'invasion de la publicité urbaine, à l'architecture fonctionnalisée ${ }^{16}$ ), jusqu'au point où il tend à devenir un principe de réorganisation du monde vécu quotidien dans sa totalité. L'échange marchand crée ses lieux, se met en scène, force les interactions. La ville se reconstruit autour de l'exigence de présenter la marchandise, de l'établir au cœur d'une vie collective intensifiée, et, ce faisant, de renforcer, d'une certaine manière, les lieux publics, de les installer au cœur de la représentation que la modernité se donne d'elle-même. Au moins la possibilité de reprises et de détournements s'ouvre-t-elle, pourrait-on ajouter dans une perspective qui ne fut cependant pas celle de l'auteur de Paris, capitale du 19e siècle.

L'approche benjaminienne connaît aujourd'hui de nombreux prolongements. David Harve ${ }^{17}$ rappelle aussi que l'origine immobilière de la crise financière de 2008 n'est pas due au hasard. Selon lui, elle nous rappelle que, à l'encontre d'une représentation commune solidement ancrée, le capitalisme actuel ne s'exprime plus seulement par la fabrication des biens industriels destinés à être consommés de façon privée, comme des cafetières ou des voitures, ni même par la fourniture de services aux personnes. Il s'exprime aussi et même beaucoup par des façons de produire l'espace ${ }^{18}$, à commencer par les milieux urbains et les infrastructures que ceux-ci réclament - donc, inévitablement, par la fabrication de lieux communs/publics, au sens géographique et architectural du terme, de lieux, de réseaux et de voies de circulation, virtuelles et réelles. Or, il est bien connu que les formes et les objets de la production revêtent une importance cruciale dans le capitalisme : toujours, le travail humain et ses résultats, les endroits où le profit se fait et comment il se fait, structurent la culture et polarisent les représentations. Il n'est donc pas indifférent, pour notre affaire, qu'une partie importante de l'activité économique (et de l'imaginaire qui s'y rattache) se cristallisent aujourd'hui autour de l'aménagement de l'espace, et, bien sûr, des déplacements dans l'espace de gens, de choses et de messages, stimulant l'imaginaire technophile de l'homme interactif et communiquant. La distance entre capitalisme et Öffentlichkeit, l'espace public au sens habermassien, qui n'est certes pas la même chose que l'espace collectif des déplacements et des présentations de soi, mais entretient tout de même quelque rapport avec lui, s'en trouve singulièrement diminuée.

13 2. Une des conséquences de l'érosion des liens hiérarchiques de type taylorien à l'intérieur de certaines organisations influentes (entreprises, agences administratives) est que la soif d'innovation concurrentielle s'exprime désormais par le fait que les idées et les 
bonnes pratiques circulent et se diffusent vite par le biais de systèmes d'évaluations et de propagation. Ce qui a de la valeur économique, ce sont, manifestement, les initiatives audacieuses et la vie communicationnelle qui leur est liée. On l'a d'ailleurs souvent remarqué $^{19}$ : la valorisation de l'interaction créatrice non-conventionnelle, historiquement antibourgeoise et même souvent anticapitaliste, est devenue déterminante pour de nombreux milieux sociaux placés désormais au centre des processus économiques de la génération du profit: des managers aux ingénieurs, en passant par toute la population des «créatifs » dans les professions liées à la production artistique, à la recherche scientifique, à l'enseignement, à la gestion des personnes et des dispositifs, aux techniques de l'information et de la communication. Il s'agit de l'expression privilégiée du fait que le néocapitalisme a organisé une sorte de captation systématique de cette grande réserve d'énergies créatrices, de besoins de participer et de réaliser, d'aspirations à la liberté qui, dans les phases antérieures de la modernité ne pouvaient pas vraiment se satisfaire et s'exprimaient même contre lui.

Par contraste avec ces anciennes phases, un des témoignages de l'influence du capitalisme sur les modes de vie et de pensée actuels consiste désormais en un énorme investissement social et culturel plus ou moins conscient de la coopération intellectuelle émancipée et autonome, implicitement perçue comme la principale source de richesses, comme une promesse infinie de développement ${ }^{20}$. Or, il est évident que cette coopération, bien qu'elle ait des causes et une portée spécifiques, vient à la rencontre de certaines exigences caractéristiques d'un espace public autonome et démocratique au sens de Habermas, c'est-à-dire un lieu où s'élabore une intelligence de soi critique du présent historique. Une inévitable synergie se met en place entre la circulation des innovations économiquement utiles ou signifiantes et la circulation des idées culturellement ou politiquement porteuses, signes d'autonomie et de vitalité d'une société civile devenue officiellement allergique au pouvoir, du moins à certaines modalités du pouvoir. Par exemple, elles empruntent les même canaux et supposent pareillement, quoiqu'en leur conférant une signification différente, une même mentalité post-traditionnelle qui convient aux dispositions de groupes souvent très proches sociologiquement.

Ainsi, des observateurs de l'économie contemporaine l'ont observé : un des moteurs du développement d'Internet après une phase initiale, celle des dernières années du siècle précédent, où il ne s'agissait que de vendre plus facilement et plus rapidement, a été la capacité de firmes à offrir sur leurs sites des services nouveaux (des conseils personnalisés, des propositions ciblées) et à piloter une nouvelle série d'échanges nouveaux entre consommateurs et vendeurs (opinions et discussions de forum sur l'usage et l'évaluation de produits, etc.) qui, dans certains cas, conduisent le consommateur à exercer une influence sur la conception des produits ${ }^{21}$. D'après les théoriciens du "capitalisme cognitif", l'effacement de la frontière entre production, circulation et consommation qui résulte de cette évolution transforme la discussion publique (qui comporte un moment critique, peut ressembler à de la critique, au sens classique et n'est donc pas un simple homonyme de la discussion politique au sens des Lumières) en source réelle de la valorisation capitaliste, en source d'enrichissement pour les entreprises. C'est donc comme si certaines provinces de l'espace public et, plus généralement, l'impulsion qui résultait de son épanouissement historique, se trouvaient désormais directement mises à profit. Un capitalisme "cognitif» qui tend à se réorganiser autour de la «production immatérielle $»^{22}$, n'est, en tout cas, plus l'autre de l'espace public de discussion et de réflexion que valorisait Habermas il y a un demi-siècle. Ce capitalisme 
est, au contraire, un gros consommateur d'échanges, de délibérations collectives, de critiques et d'initiatives qui meublent et agrandissent le monde public. À certains niveaux, il y a inévitablement interpénétration.

16 3. Dans L'espace public, Habermas mobilisait une critique de la culture contemporaine largement inspirée par les motifs adorniens. Parler d'« industrie culturelle», montrer comment les discours et les images qui nourrissent la pensée et l'imaginaire collectifs étaient soumis à des impératifs de rentabilité capitaliste qui en dégradaient inévitablement le contenu, c'était montrer qu'aucune vitalité politique authentique ne peut venir animer nos sociétés en s'appuyant sur eux. Du point de vue habermassien, la gravité du dévoiement de la culture provient du fait que celle-ci est censée alimenter l'espace public et bénéficier en retour de sa vitalité. D'où vient l'impression - qui modifie forcément les conclusions habermassiennes - que nous ne sommes plus tout à fait aujourd'hui à l'âge où Adorno pouvait mettre en cause à la fois l'application choquante au domaine de la culture de formes, organisationnelles comme techniques, liées à la grande industrie, ainsi que la diffusion d'idéologies politiquement suspectes qu'elle rendait possible?

D'abord, du fait que la capacité d'absorption de la critique et la vitesse avec laquelle elle s'effectue se sont accrues dans des proportions stupéfiantes. Il ne peut donc plus seulement s'agir de dénoncer les déformations et les limitations que les puissances occultes, sociales, politiques ou économiques, font subir à l'espace public. La critique des altérations que subissent les normes immanentes à un espace public libéré n'a plus besoin des philosophes ou des sociologues; elle est largement assurée dans cet espace public, entrainant, d'ailleurs, une course de vitesse entre forces contradictoires. Comme au terme d'une accélération spectaculaire du rythme de l'histoire, la critique et ses cibles ont été endogénéisées ensemble, comme deux forces motrices du medium lui-même. C'est ainsi que, au jour le jour, Internet vit de l'ingéniosité illimitée des puissances commerciales, plus généralement, des stratégies de communication menées par les forces économiques, sociales ou politiques déchainées, et des habiles contre-feux qu'on leur oppose : media alternatifs, campagnes de dénonciation, enquêtes et contre-enquêtes, débats d'idées. Par exemple, on y voit à la fois une explosion sans précédent de la pollution publicitaire et l'élaboration d'une puissante critique de la publicité et du consumérisme.

18 Ensuite et surtout ce qui a changé la situation, c'est l'importance économique énorme acquise à l'échelle internationale par la production des œuvres audiovisuelles ${ }^{23}$, qui jouent un rôle paradigmatique sur le plan de l'organisation du travail et du marketing et aussi dans un processus mondialisé d'éducation des sensibilités et des conduites qui conditionne, via le consumérisme, le développement futur du capitalisme en fournissant sa principale base de légitimation. On se situe là à la pointe du dynamisme économique et non plus à sa traine. La conséquence est que, contrairement à un enseignement que l'on pourrait tirer de Habermas, ici proche d'Adorno, l'industrie de la culture ne constitue pas une force agressive dont le principe contredirait la liberté et l'égalité censées régner dans un espace public digne de ce nom qui transcenderait l'ordinaire d'une vie sociale livrée à la plate domination ou à la routine étouffante. Certes, elle comporte un aspect idéologique et exploitatif au sens où, par exemple, le cinéma hollywoodien s'empare assez souvent des préoccupations écologistes et sanitaires qui se développent dans l'espace public pour les tirer dans le sens d'un apocalyptisme viril, autoritaire et ethnocentrique plus ou moins délirant ${ }^{24}$. Mais, de façon générale, il faudrait plutôt voir l'industrie 
culturelle comme la manière dont le capital, non content de subsister à ses marges et de l'utiliser, tente de s'installer dans un milieu accueillant qui est aussi celui de «l'espace public ", au sens des Lumières, pour y jouer sa propre partition et bénéficier d'un environnement favorable, voire tente de le constituer dans ses formes comme dans ses contenus. Il n'y a donc pas seulement interpénétration du «travail immatériel » et de l'espace public, mais surtout une sorte de tendance active à promouvoir et à parasiter systématiquement ce dernier, d'un même mouvement. En effet, réalisant caricaturalement l'idée foucaldienne d'une opinion publique instituée et promue par le pouvoir politique comme son partenaire ${ }^{25}$, cette industrie tente de produire ce que la société civile, émancipée de ses tutelles traditionnelles, voit et pense. Car sans qu'il soit besoin de croire que les gens sont manipulés du matin au soir et se réduisent désormais à de simples jouets des intérêts des multinationales, il faut bien constater que, sous cette forme, elle exerce désormais une influence considérable sur la constitution et le devenir mondialisé des formes de vie, à un âge où l'arrière-plan traditionnel et bourgeois des formes sociales, encore déterminant dans les phases historiques antérieures, devient beaucoup moins prégnant.

La fonction que remplit cette tentative d'installation est facile à identifier. Il ne s'agit pas de divertissement ou d'opium du peuple, ni du contrôle de l'opinion au sens classique d'une emprise du pouvoir politique sur les cerveaux. Il s'agit plutôt de la fabrication économiquement rentable d'un consentement tranquille, en l'occurrence de la fabrication d'une évidence cruciale, celle selon lesquelles les vies humaines sont vouées à la marchandise et au marché. Car ce que «vend » l'industrie culturelle aujourd'hui, au sens des contenus et des formes, c'est d'abord un a priori, celui qui fonde l'esprit du capitalisme néolibéral. Telle est même probablement sa fonction majeure. Ainsi, le héros hollywoodien aujourd'hui, c'est souvent (pas toujours!) celui qui, entre autres choses et sous une forme plus ou moins métaphorique, déploie aisance et naturel, jusqu'à la virtuosité, dans l'univers technique, commercial et concurrentiel ; c'est celui pour qui la consommation marchande, ainsi que les dispositions psychiques qui vont dans le sens du rapport marchand à soi, à autrui et au monde ont été manifestement intériorisées sans accrocs et peuvent donc être exprimées passionnément. Ainsi, sous des modalités très variées, les dispositions néolibérales forment aujourd'hui le centre de gravité de l'industrie culturelle: les représenter, c'est leur conférer une aura de légitimité, les installer au centre des représentations collectives, les rendre capable d'essayer de donner le ton à ce qui se passe dans l'espace public. Dans ces conditions, plus besoin, pour conformer les individus, de contrecarrer la dynamique immanente de l'espace public.

Ainsi, sur fond de l'a priori néolibéral, les puissances aliénantes, classes dominantes ou entreprises influentes, recourent généralement à une technique très sûre, qui constitue probablement le principe de toutes celles qui se développent dans l'industrie culturelle, consiste à saturer l'espace public médiatique de flux ininterrompus de sons, d'images et de paroles. Exploitant et alimentant sans cesse l'espace public en même temps, respectant formellement son mode de fonctionnement en l'enrichissant d'une façon fantastique, ces puissances du soft power n'ont besoin que d'un très petit et très discret commun dénominateur pour pouvoir être sélectionnés : il faut que, à un niveau ou à un autre, le bien marchand en général et la vie commandée par le fétichisme de la marchandise y apparaissent ou soient suggérés, même de loin, comme formant la condition normale de l'existence, l'horizon indépassable de l'action. En fonction de cet impératif souple, ce qui n'était qu'embryonnaire à l'époque où écrivait Habermas a pris le visage d'une force 
prodigieuse, fabricatrice d'une culture globale dans laquelle nous baignons : la force qui, tout en élargissant de façon gigantesque le périmètre de l'espace public (grâce aux développement des techniques d'information et de communication), engorge sans cesse ses canaux par de la pseudo-connaissance du présent, par de la pseudo-pluralité, par de la pseudo-information, par de pseudo-débats, par de la pseudo-transparence, par de la pseudo-critique, par de la pseudo-réflexivité, bref par de la pseudo-autonomie publique autant de phénomènes que les conceptions classiques de l'« idéologie " ne permettent guère d'appréhender ${ }^{26}$. Étant entendu que les ressemblances et les continuités entre formes authentiques et inauthentiques sont nombreuses et que personne ne peut décemment prétendre disposer du critère univoque permettant d'opérer des partages certains et définitifs. Dans l'industrie culturelle contemporaine, dont la présentation de l'individu néolibéral forme le pôle de référence caché, l'Öffentlichkeit se trouve donc tout à la fois sollicitée, constituée et distordue.

Les conséquences d'un tel état de fait sont faciles à deviner. Il est banal de dire que, aujourd'hui, ce n'est plus la rareté de l'information, mais sa surabondance qui est problématique, parce que celle-ci anesthésie la réflexion, provoquant une sorte de sidération générale qui finit par brouiller les différences et les hiérarchies, par tout niveler, le massacre de masse et le match de foot, les grands mouvements sociaux et les caprices des stars. C'est que, en effet, le régime de la zone grise devient la règle, à commencer par celle qu'impose la gigantesque galaxie qui conglomère désormais information, divertissement, culture, apprentissage (learning), art, savoir, communication, publicité - tous rendus homogènes, dans beaucoup de leurs parties, par les contraintes du fonctionnement marchand, ainsi que par les valeurs et les formes que celui-ci secrète spontanément. Il est également banal de dire que les médias d'information du mainstream se situent au cœur de cette transformation. À ces banalités, il nous suffira donc d'ajouter que, dans ce monde de la production internalisée du consentement, qui, selon les cas, sollicite et met en scène les formes de vie et d'individualité acquises aux besoins du capital, l'espace public est devenu, aux conditions précisées, à la fois une ressource exploitable et un milieu quasi productible, en tout cas l'une des niches écologiques les plus accueillantes du néocapitalisme, pourvu qu'on la soutienne un peu.

Encore une fois, cela ne signifie pas qu'il soit devenu impossible ou vain de faire des différences entre la bonne et la mauvaise discussion, la bonne et la mauvaise information, entre la culture purement commerciale et la culture intéressante, authentiquement créative et/ou riche de sens, entre l'enquête instructive et la simple manipulation, entre la participation sincère à la réflexion collective et la stratégie de communication sans foi ni loi (ou le bavardage insignifiant); ni qu'il soit devenu impossible de tirer des conséquences pratiques de ce type de différences. Bien des gens font ces différences, des gens pour lesquels la lutte autour des valeurs typiques de l'espace public (celles qui gravitent autour de la constitution d'une conscience lucide du présent historique et d'une délibération équitable, utile, tournée vers l'avenir) gardent heureusement leur sens. Cela signifie plutôt que tous ces éléments contradictoires, à force de coexister et d'interagir, finissent souvent par se ressembler, par s'imiter, par se fondre les uns dans les autres, par s'associer selon des styles communs, et que les partages et les découpages s'avèrent, pour ces raisons, souvent aussi difficiles à opérer que relatifs. Notre monde post-habermassien, sa culture en tout cas, sont faits comme cela ; ils ne sont pas plus ni moins atroces, de ce point de vue, que ceux qui les ont précédé et c'est bien d'eux, en tout cas, qu'il faut partir. 
Car, pour une grande part, les fonctions cognitives, sociales et politiques de l'espace public ne peuvent désormais s'exercer qu'à l'intérieur de ce milieu incommode que le néocapitalisme entretient pour ses propres besoins; pour une grande part, elles ne peuvent s'exercer qu'en jouant sur les contraintes et en exploitant les ambigüités propres à ce milieu.

\section{NOTES}

1. J. Habermas, Strukturwandel der Öffentlichkeit. Untersuchungen zu einer Kategorie der bürgerlichen Gesellschaft [1962], Francfort, Suhrkamp, 1990. En traduction française : L’Espace public, Paris, Payot, 1995.

2. Si R. Chartier (Les Origines culturelles de la Révolution française, Paris, Seuil, 1990) est encore très proche de l'esprit des analyses habermassiennes, les travaux ultérieurs se font, en général, plus nuancés, parfois très critiques. Voir, sur l'exemple des études médiévales, P. Boucheron et N. Offenstadt, L'Espace public médiéval, Paris, PUF, 2011.

3. Citons trois exemples importants dans l'historiographie de langue française actuelle : $\mathrm{S}$. Van Damme, Paris, capitale philosophique, Paris, O. Jacob, 2005 ; A. Lilti, Le Monde des salons, Paris, Fayard, 2005 ; S. Landi, Naissance de l'opinion publique dans l'Italie moderne, PU de Rennes, 2006.

4. L'idée (fort problématique, en fait) d'une unité intellectuelle profonde des Lumières (dont le kantisme résumerait et sublimerait l'apport) est, par exemple, au cœur de l'ouvrage célèbre de Cassirer, Die Philosophie der Aufklärung, paru en 1932. On peut penser que le livre de Habermas, dans un autre ordre, exprime une conviction très proche et que cette conviction exerce une forte influence sur la manière dont s'y écrit le récit de l'évolution de l'espace public en Occident.

5. Une partie de la réflexion philosophique actuelle part bien de l'idée que, avec l'initiative consistant à parler à quelqu'un de quelque chose (ce que Habermas appelle «l'action communicationnelle »), un type très particulier de séquence d'événements peut s'enclencher, auquel nous avons intérêt à rattacher notre volonté d'expliciter des notions générales et opaques comme celles de "langage ", de "raison", de "rapport social » ou même de "sujet». Voir surtout R. Brandom, Making it explict, Harvard UP, 1994.

6. Les arguments les plus convaincants en faveur du contextualisme en philosophie du langage ont été articulés par Fr. Recanati : Le sens littéral, Paris, L'Éclat, 2007.

7. C'est un des points forts du pragmatisme états-unien que d'avoir insisté sur cet aspect. Voir R. Frega, John Dewey et la philosophie comme épistémologie de la pratique, Paris, L'Harmattan, 2009.

8. Voir, pour une reprise récente, A. Honneth, Das recht der Freiheit, Berlin, Suhrkamp, 2011, p. 470 et suiv.

9. Voir Théorie de l'agir communicationnel, Paris, Fayard, 1987. L'analyse semble aussi valoir pour le cas de la domination politique et administrative. L'idée classique du pouvoir qui cherche à étouffer la discussion entre citoyens parce qu'il sent qu'elle le remettra inévitablement en cause a perdu aujourd'hui l'essentiel de sa portée, à mesure que la négociation apparaissait progressivement comme une forme fondamentale de gouvernement. Certes, en principe, la distinction entre une délibération, par définition imprévisible, capable de renverser les cadre fixés, et une négociation sous contraintes, en général lourdement institutionnalisée, est très 
facile à faire. Mais il reste qu'un pouvoir qui, inversant la logique habituelle du conservatisme, réclame la mobilisation des énergies en vue du changement et organise pour cela des négociations en cascade, devenues sources apparentes de toute légitimation, exerce une domination d'un genre nouveau. Il s'installe dans les parages de la délibération, dans les parages de la démocratie non-représentative, et il demande à chacun d'assumer un rôle qui ressemble de manière troublante à celui du citoyen délibératif, même s'il est évident qu'il en diffère profondément. Comme dans le cas du capitalisme, on voit que les puissances sociales potentiellement aliénantes, politiques ou économiques, pour reprendre des catégories traditionnelles, n'apparaissent plus toujours comme extérieures, opposées aux manifestations de l'autonomie, de la discussion, de la création. Loin de se borner à les "récupérer ", ces puissances les suscitent, les encadrent. Certes, les contraintes et les déformations que ces puissances exercent sur l'expression de dites tendances restent repérables, mais elles n'ont plus la netteté d'autrefois.

10. Notons cependant que le propre des théories du capitalisme cognitif est de tabler sur une fusion totale, déjà accomplie, de l'univers de la production et de l'espace public. Ici, nous supposerons seulement l'existence d'une continuité.

11. Voir Th. Paquot, L'Espace public, Paris, La Découverte, 2009.

12. M. Davis, City of Quartz. Los Angeles, capitale du futur, Paris, La Découverte, 2000.

13. Voir aussi B. Bégout, Zéropolis. L'expérience de Las Vegas, Paris, Allia, 2002.

14. Voir R. Sennett, Les Tyrannies de l'intimité, Paris, Seuil, 1979. Les questions contemporaines liées à la propriété intellectuelle suscitent des analyses semblables. Voir par exemple D. Bensaïd, Les Dépossédés, Paris, La Fabrique, 2007.

15. W. Benjamin, Paris, Capitale du $19^{\mathrm{e}}$ siècle, Paris, Cerf, 1993.

16. Voir l'analyse de l'architecture contemporaine chez F. Jameson, Le Postmodernisme ou la logique culturelle du capitalisme tardif, Beaux-Arts de Paris, 2007.

17. The Enigma of Capital, Londres, Profile Books, 2010.

18. Selon un concept qui fut d'abord élaboré par H. Lefebvre. Voir La Production de l'espace [1974], Paris, Anthropos, 2000.

19. L. Boltanski È. Chiappello, Le Nouvel esprit du capitalisme, Paris, Gallimard, 1999.

20. M. Lazzarato, Puissances de l'invention, Paris, Empêcheurs de penser en rond, 2002.

21. Voir T. Terranova, « New Economy, Financialization and Social Production in the Web 2.0 » in

A. Fumagalli and S. Mezzadra (ed.), Crisis in the Global Economy, Los Angeles, Semiotext(e), 2010, p. 153-171.

22. Y. Moulier Boutang, Le Capitalisme cognitif, Paris, Amsterdam, 2009.

23. Rappelons par exemple que le film Avatar a généré en quelques mois environ trois milliards de dollars US de recettes (contre un coût de 387 millions de dollars, marketing compris : http:// en.wikipedia.org/wiki/Avatar_\%282009_film\%29). L'ampleur de tels profits et la rapidité avec laquelle ils se produisent donnent beaucoup à penser à propos de l'évolution du capitalisme actuel.

24. Voir, par exemple : The Day after Tomorrow, R. Emmerich, 2004 ; I am Legend, F. Lawrence, 2007 ; et, de nouveau, Avatar, J. Cameron, 2009.

25. À l'hypothèse idéaliste selon laquelle, à un moment, s'est produit ce miracle que fut l'émergence d'une opposition pure au pouvoir, il faudrait substituer, d'après Foucault, l'approche selon laquelle cette opposition forma d'abord l'un des éléments constitutifs d'une nouvelle configuration de pouvoir, post-absolutiste, où l'État et les puissances qui s'y rattachent ont eu besoin de s'appuyer sur une société civile active et de la (re)connaitre. La fiction de l'«opinion publique » ne serait donc pas seulement une nouvelle figure de la dépossession politique, mais bien aussi le corrélat d'une forme de gouvernement déterminée. Voir M. Foucault, Sécurité, territoire, population, Paris, Gallimard/Seuil, 2004, p. 283-284. On trouve une systématisation approfondie de cette hypothèse à propos du cas français chez D. Reynié : Le Triomphe de 
l'opinion publique, Paris, O. Jacob, 1998. Pour la manière dont les décisions judiciaires contribuent à définir l'espace public dans les sociétés contemporaines, voir M. Iacub, De la pornographie en Amérique, Paris, Fayard, 2010.

26. Habermas lui-même notait dès la fin des années 60 le caractère bien trop étroit de la conception traditionnelle (jeune marxienne) de l'idéologie face aux évolutions du capitalisme contemporain. Voir La Science et la technique comme « idéologie », Paris, Gallimard, 1990.

\section{AUTEUR}

\section{STÉPHANE HABER}

Codirecteur du Congrès Marx International, professeur et directeur du département de philosophie à Paris Nord. Il a introduit les principaux arguments et livre d'Axel Honneth en France et récemment publié L'homme dépossédé, CNRS éditions, 2010. 